\title{
Intradural Intramedullary Primary Spinal Melanocytoma: A Rare Case Report
}

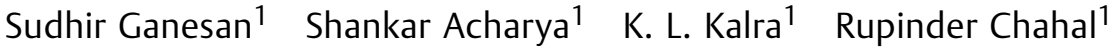 \\ ${ }^{1}$ Ortho Spine Department, Sir Ganga Ram Hospital, New Delhi, India \\ Address for correspondence Dr. Sudhir Ganesan, MBBS, DNB, \\ Indian J Neurosurg 2017;6:55-58. \\ MNAMS, FNB, Ortho Spine Department, Sir Ganga Ram Hospital, Old \\ Rajinder Nagar, New Delhi 110060, India \\ (e-mail: sudhiraxon@gmail.com).
}

\begin{abstract}
Keywords

- intradural intramedullary melanocytoma

- pigmented tumor

Spinal melanocytomas are rare pigmented tumors of the central nervous system commonly affecting females in their fifth decades. It has been reported in various locations with intradural intramedullary being the rarest. Patients can present with varied symptomatology from mild backache to frank myelopathy. Here we report a case of 34-year-old man with no medical comorbidities with vague backache associated with numbness of anterior aspect of right thigh for 1 year aggravated by activities and relieved by rest. His neurologic examination revealed normal power and sensation in both lower limbs except for decreased temperature sensation in right L2 dermatome. MRI of dorsolumbar spine revealed a well-defined lesion that was hyperintense in T1- and hypointense in T2-weighted image at D11-12 region with uniform bright enhancement in gadolinium-enhanced $\mathrm{T} 1$ image. The patient underwent laminectomy of D11 and D12, and an en bloc excision was done. Diagnosis of primary intradural intramedullary melanocytoma was made from histopathologic examination. Spinal melanocytomas should be considered as a differential diagnosis for intradural space-occupying lesions. MRI remains the investigation of choice for diagnosis, but histopathologic examination is required to confirm and differentiate from other pigmented tumors and malignant melanomas. Total surgical excision of the tumor remains the standard treatment with radiotherapy reserved for cases of incomplete excision and recurrences.
\end{abstract}

\section{Introduction}

Spinal melanocytomas, first described in $1972,{ }^{1}$ are rare pigmented tumors of the central nervous system commonly affecting females in their fifth decades. It commonly occurs in the posterior fossa, but spinal melanocytomas have also been reported in various locations with intradural intramedullary lesions being the rarest. Patients can present with varied symptomatology from mild backache, radiculopathy, to frank myelopathy like other intradural space-occupying lesions. ${ }^{1}$ They are generally benign but can be locally aggressive and very rarely can undergo malignant transformation. Magnetic resonance imaging (MRI) remains

received

December 28, 2015

accepted after revision

February 10, 2016

published online

July 26, 2016
DOI http://dx.doi.org/

10.1055/s-0036-1584589. ISSN 2277-954X. the investigation of choice for diagnosing melanocytomas, but histopathologic examination of the specimen is mandatory for confirming the diagnosis. ${ }^{2}$ We report a rare case of a 34-year man with an intradural intramedullary primary spinal melanocytoma who presented with vague back pain and numbness in right $\mathrm{L} 2$ dermatome without any other findings.

\section{Case Report}

A 34-year-old man with no medical comorbidities was admitted in our hospital with vague backache associated with numbness of anterior aspect of the right thigh for (c) 2017 Neurological Surgeons' Society of India

License terms

(c) $9 \ominus \$$ 
1 year aggravated by activities and relieved by rest. The pain was worse at night and was not associated with any weakness of limbs. There was no loss of appetite or weight loss. He had no fever or other joint pain. He was treated conservatively elsewhere with analgesics and physiotherapy but had no relief. On neurologic examination, he had grade 5 power and normal sensation in both lower limbs except for decreased temperature sensation in right L2 dermatome. Reflexes were normal and plantars were flexors bilaterally. His blood investigations and X-ray were within normal limits. X-ray lumbosacral spine was normal and MRI of the dorsolumbar spine revealed a well-defined lesion that was hyperintense in T1-weighted image and hypointense in T2-weighted image at D11-12 region (-Figs. 1-4. The lesion showed uniform bright enhancement in gadoliniumenhanced T1 image (-Fig. 5). Complete excision of the lesion was planned. The patient underwent laminectomy of D11 and D12. The dura was incised and the lesion was seen as a dark black mass with pial invasion (-Fig. 6). The lesion was demarcated from the surrounding tissue and an en bloc excision was done. Postoperative period was uneventful. Drain was removed and the patient was mobilized on the second day. Histopathologic examination revealed spindle cells arranged in bundles and fascicles pigmented by melanin with no cellular atypia or increased mitosis. The tumor cells were positive for HMB 45 and negative for S-100 and EMA. Proliferative index (Ki67) was low $(<1 \%)$. The patient was subjected to a detailed dermatologic and ophthalmologic examination to rule out melanotic lesions in other sites. Whole-body FDG-PET CT (fluorodeoxyglucose positron emission tomography-computed tomography) was done, which was negative for any uptake in other sites and a diagnosis of primary spinal intradural intramedullary melanocytoma was made. The patient did not receive radiotherapy or chemotherapy postoperatively. He had complete recovery of the numbness and returned to his

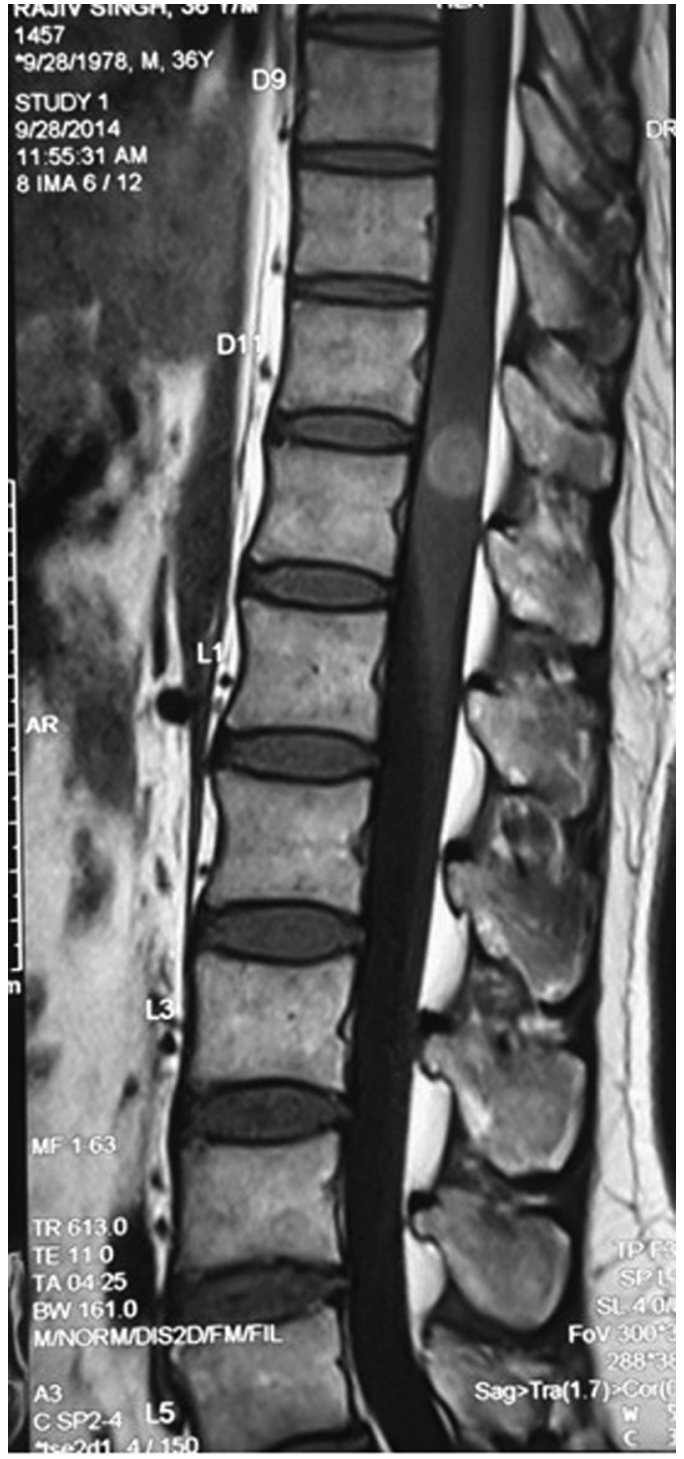

Fig. 1 Sagittal T1-weighted MRI of dorsolumbar spine showing a well-defined hyperintense lesion at D11-12 region.

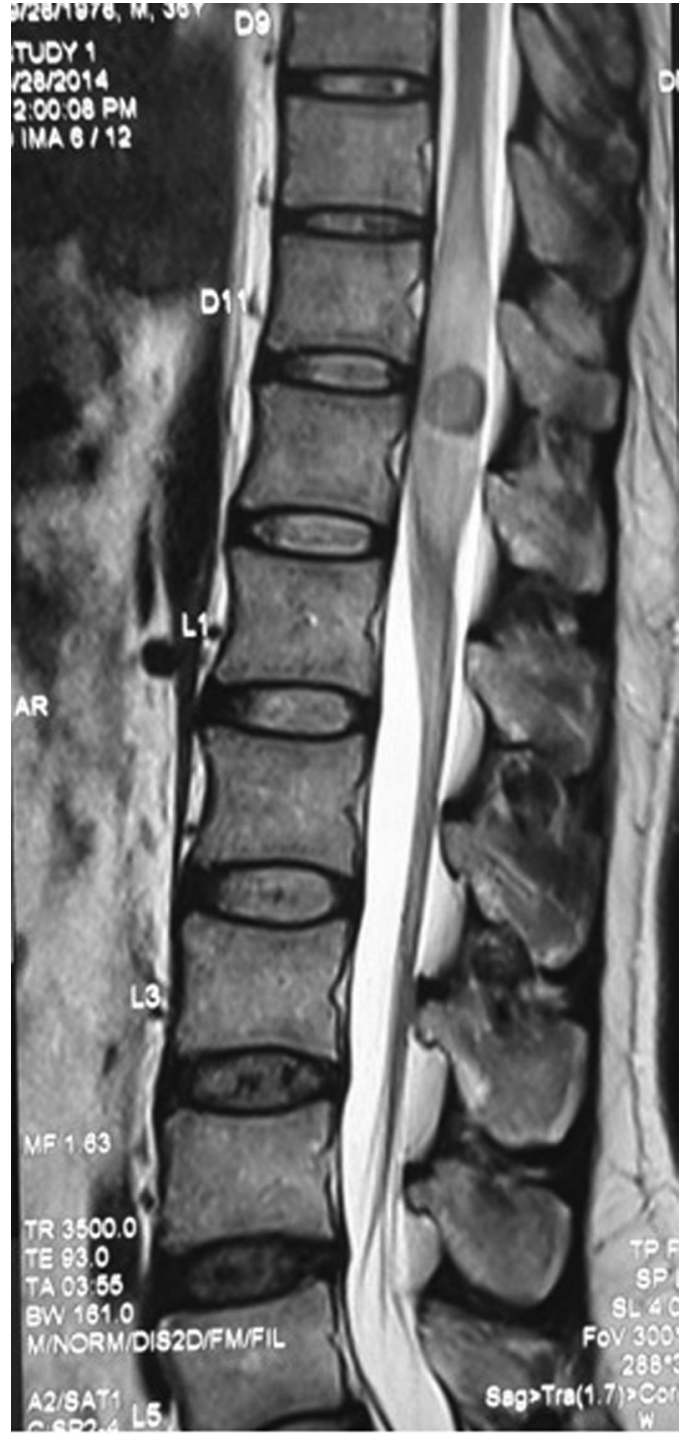

Fig. 2 Sagittal T2-weighted MRI of dorsolumbar spine showing a well-defined hypointense lesion at D11-12 region. 


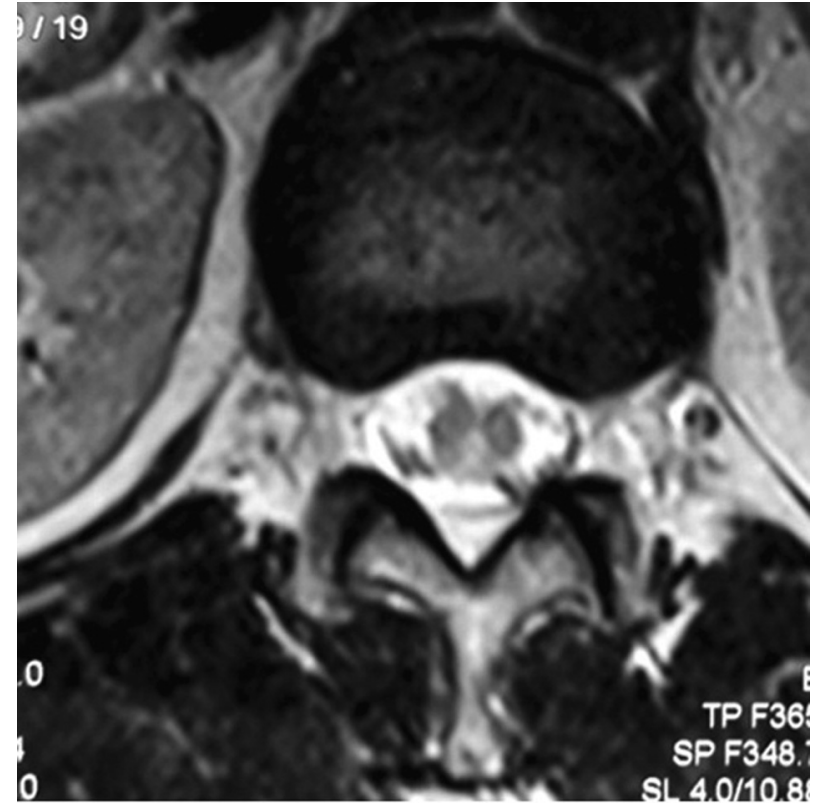

Fig. 3 Axial T2-weighted image showing a well-defined hypointense lesion at D11-12 region.

normal activities with no complaints.

\section{Discussion}

Primary spinal melanocytoma is a rare entity accounting for very few cases in the literature since the first electron microscopic description by Limas and Tito ${ }^{1}$ in 1972 who differentiated the benign melanocytomas from the tumors originating from meningothelial fibroblasts. The types of melanotic lesions of the central nervous system as described by World Health Organization include diffuse melanocytosis, melanomatosis, melanocytoma, and malignant melanoma. Brat et al classified melanotic lesions into low-,

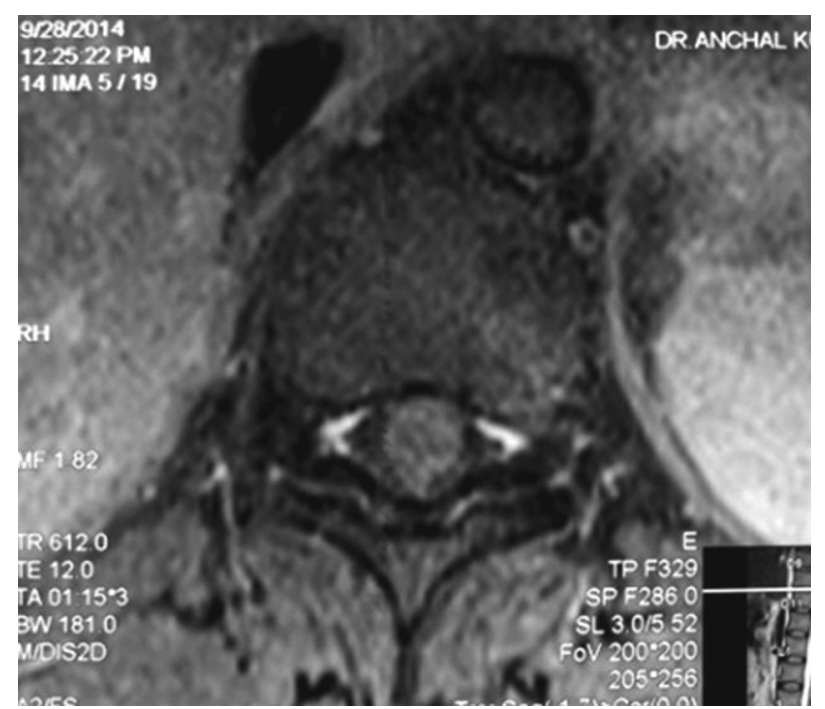

Fig. 4 Axial T1-weighted image with a well-defined hyperintense lesion at D11-12 region.

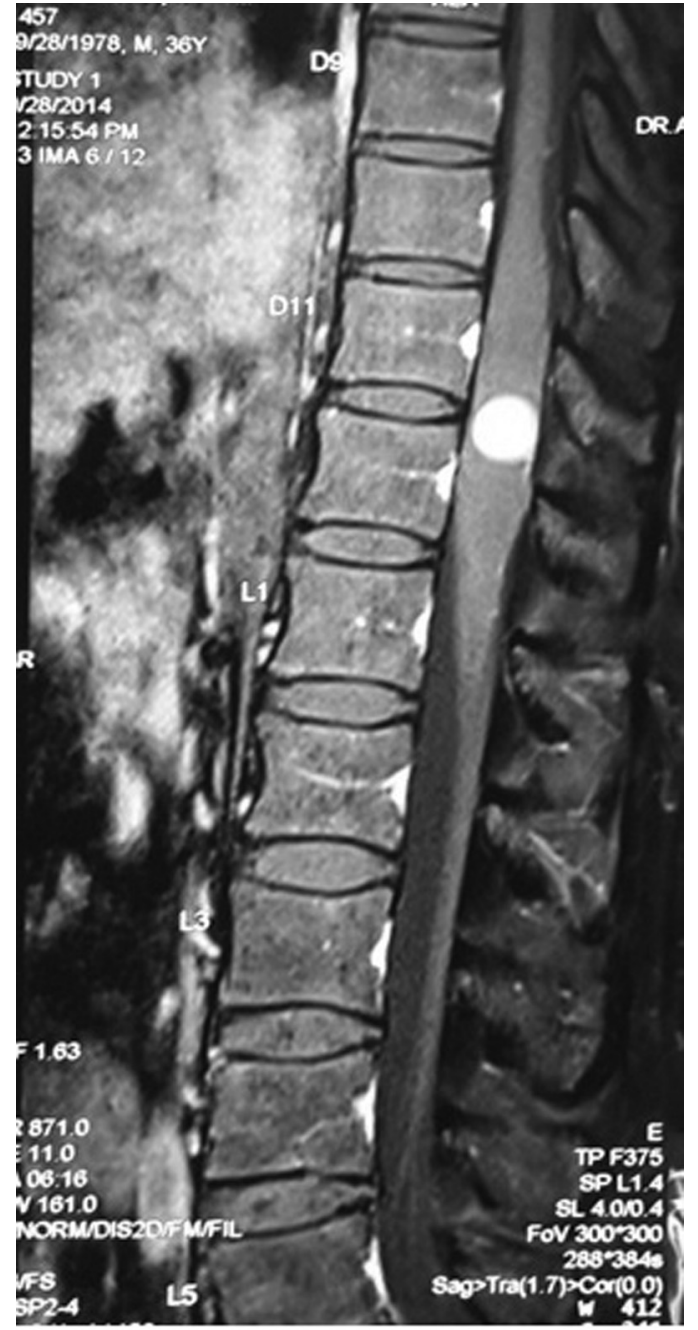

Fig. 5 Contrast-enhanced sagittal T1-weighted image showing uniform bright enhancement of the lesion.

intermediate-, and high-grade based on the histologic differentiation. ${ }^{3}$ Posterior fossa is the most common region to be affected, but cases of cervical and lumbar spinal melanocytomas have also been reported. ${ }^{3}$ Approximately one-third of melanocytomas arise from the superior spinal cord. Extramedullary lesions are most common with only a few case reports of intramedullary location. ${ }^{3}$ They originate

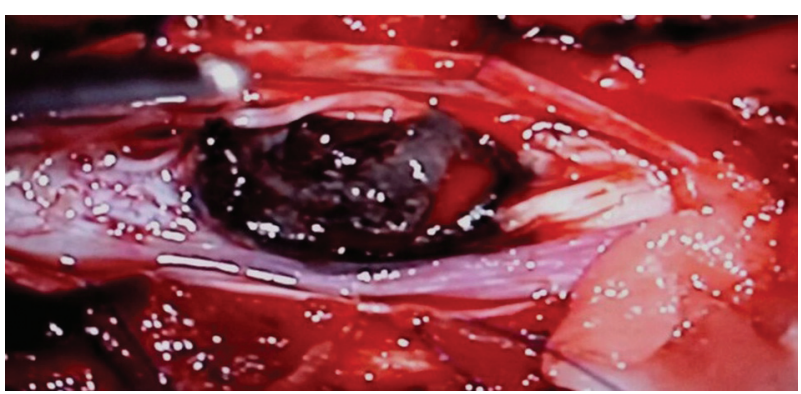

Fig. 6 Intraoperative image showing dark black mass with pial invasion. 
from the melanocytes along the neural crest with typical occurrence in the leptomeninges. ${ }^{4}$ Females are more commonly affected than males with a peak incidence in the fifth decade. ${ }^{4}$ The clinical presentation of these patients vary from minimal vague symptoms to myelopathy mimicking other intradural space-occupying lesions. ${ }^{1}$ Melanocytomas, though considered benign, can undergo malignant transformation into melanomas.

MRI remains the gold standard for the diagnosis of spinal tumors and it is the investigation of choice for spinal melanocytoma. It typically appears as a hyperintense lesion in T1-weighted MR images and hypo- or isointense in T2-weighted MR images. ${ }^{2,5}$ The tumor enhances uniformly in gadolinium contrast-enhanced T1-weighted MR images. ${ }^{2,5}$ Though this is the classic appearance of melanocytoma, it can vary depending on the melanin content, presence of fat, and hemorrhages. Differential diagnosis includes schwannomas, meningiomas, and melanomas. Hence, differentiating melanocytoma from other spinal tumors is difficult by MRI, and histopathologic examination is mandatory for confirming the diagnosis. ${ }^{2}$ Our case had the typical appearance of spinal melanocytoma that was confirmed by histopathologic examination.

On gross examination, melanocytomas appear as wellencapsulated dark brown to black nodule. The histologic features include spindle or epithelioid cells with intracytoplasmic melanin pigment arranged in fascicles, whorls, sheets, or bundles surrounded by a fine network of reticulin fibers. Tight clustering of the tumorous cells and prominent nucleoli have also been reported. ${ }^{3,6}$ Histologically, melanocytoma can be differentiated from melanoma by the presence of nuclear atypia, mitotic figures, necrosis, hemorrhage, and microvascular invasion and a high proliferation index $\left(K_{i} 67>5 \%\right)$ in the latter. Immunohistochemistry reveals a positive staining for HMB-45, S100 protein, vimentin, and a negative staining for epithelial membrane antigen (EMA), glial fibrillary acidic protein, and neuron-specific enolase. ${ }^{6}$ The tumor cells in our case were positive for HMB 45 but negative for S-100 and EMA, and the proliferative index $\left(\mathrm{K}_{\mathrm{i}} 67\right)$ was less than $1 \%$.

Complete surgical resection of the tumor has been the treatment of choice for intradural spinal melanomas. ${ }^{7,8}$ Though melanocytoma is a benign tumor, it can be locally aggressive and cases of late recurrence due to incomplete excision have been reported by few authors. ${ }^{7}$ The role of radiotherapy and chemotherapy is very controversial in literature with no definite guidelines. Kurita et $\mathrm{al}^{9}$ have reported the beneficial effect of radiotherapy in melanocytomas. The dosage depends on various factors such as the location, size, patient tolerance, etc. Radiotherapy is usually preferred in cases of incomplete resection of the tumor. The role of chemoimmunotherapy has been emphasized in a case report of thoracic melanocytoma by Verma et $\mathrm{al}^{10}$ in which they showed a remission of 15 months. However, long-term studies confirming the same are required. Radiotherapy was not given to our patient as we could excise the tumor en bloc.

Melanocytomas are benign tumors and tend to show a slow progression rate compared with melanomas. Unlike melanomas, melanocytomas have good prognosis with increased life expectancy. However, these patients require follow-up clinical examination and MRI as the local recurrence rate can reach up to $50 \%$.

\section{Conclusion}

Spinal meningeal melanocytomas, benign rare pigmented tumors, can be locally aggressive with varied clinical presentation. MRI remains the investigation of choice for diagnosis, but histopathologic examination is required to confirm and differentiate from other pigmented tumors and malignant melanomas. Total surgical excision of the tumor remains the standard treatment of choice with radiotherapy reserved for cases of incomplete excision and recurrences.

\section{Conflict of Interest}

None.

Funding

No funding received.

\section{References}

1 Limas C, Tio FO. Meningeal Melanocytoma ("Melanotic meningioma"). Its melanocytic origin as revealed by electron microscopy. Cancer 1972;30:1286-1294

2 Uematsu Y, Yukawa S, Yokote H, Itakura T, Hayashi S, Komai N. Meningeal melanocytoma: magnetic resonance imaging characteristics and pathological features. Case report. J Neurosurg 1992;76(4):705-709

3 Brat DJ, Giannini C, Scheithauer BW, Burger PC. Primary melanocytic neoplasms of the central nervous systems. Am J Surg Pathol 1999;23(7):745-754

4 Shanthi V, Ramakrishna BA, Bheemaraju VV, Rao NM, Athota VR. Spinal meningeal melanocytoma: a rare meningeal tumor. Ann Indian Acad Neurol 2010;13(4):308-310

5 Czarnecki EJ, Silbergleit R, Gutierrez JA. MR of spinal meningeal melanocytoma. AJNR Am J Neuroradiol 1997;18(1):180-182

6 Ibáñez J, Weil B, Ayala A, Jimenez A, Acedo C, Rodrigo I. Meningeal melanocytoma: case report and review of the literature. Histopathology 1997;30(6):576-581

7 Ali Y, Rahme R, Moussa R, Abadjian G, Menassa-Moussa L, Samaha E. Multifocal meningeal melanocytoma: a new pathological entity or the result of leptomeningeal seeding? J Neurosurg 2009;111(3):488-491

8 Horn EM, Nakaji P, Coons SW, Dickman CA. Surgical treatment for intramedullary spinal cord melanocytomas. J Neurosurg Spine 2008;9(1):48-54

9 Kurita H, Segawa H, Shin M, et al. Radiosurgery of meningeal melanocytoma. J Neurooncol 2000;46(1):57-61

10 Verma DS, Spitzer G, Legha S, McCredie KB. Chemoimmunotherapy for meningeal melanocytoma of the thoracic spinal cord. Report of a case. JAMA 1979;242(22):2435-2436 